\title{
Inducible nitric oxide synthase gene deficiency counteracts multiple manifestations of peripheral neuropathy in a streptozotocin-induced mouse model of diabetes
}

\author{
I. Vareniuk • I. A. Pavlov • I. G. Obrosova
}

Received: 3 June 2008 / Accepted: 22 July 2008 / Published online: 19 September 2008

(C) Springer-Verlag 2008

\begin{abstract}
Aims/hypothesis Evidence for the importance of peroxynitrite, a product of superoxide anion radical reaction with nitric oxide, in peripheral diabetic neuropathy is emerging. The role of specific nitric oxide synthase isoforms in diabetesassociated nitrosative stress and nerve fibre dysfunction and degeneration remains unknown. This study evaluated the contribution of inducible nitric oxide synthase (iNOS) to peroxynitrite injury to peripheral nerve and dorsal root ganglia and development of peripheral diabetic neuropathy. Methods Control mice and mice with iNos (also known as Nos2) gene deficiency $\left(\mathrm{iNos}^{-1-}\right.$ ) were made diabetic with streptozotocin, and maintained for 6 weeks. Peroxynitrite injury was assessed by nitrotyrosine and poly(ADP-ribose) accumulation (immunohistochemistry). Thermal algesia was evaluated by paw withdrawal, tail-flick and hot plate tests, mechanical algesia by the Randall-Selitto test, and tactile allodynia by a von Frey filament test.

Results Diabetic wild-type mice displayed peroxynitrite injury in peripheral nerve and dorsal root ganglion neurons. They also developed motor and sensory nerve conduction velocity deficits, thermal and mechanical hypoalgesia, tactile allodynia and $\sim 36 \%$ loss of intraepidermal nerve fibres. Diabetic $\mathrm{iNos}^{-/}$mice did not display nitrotyrosine and poly(ADP-ribose) accumulation in peripheral nerve, but were not protected from nitrosative stress in dorsal root ganglia. Despite this latter circumstance, diabetic $i \mathrm{Nos}^{-1-}$ mice preserved normal nerve conduction velocities. Small-
\end{abstract}

I. Vareniuk · I. A. Pavlov · I. G. Obrosova $(\bowtie)$

Pennington Biomedical Research Center,

Louisiana State University System,

6400 Perkins Road,

Baton Rouge, LA 70808, USA

e-mail: obrosoig@pbrc.edu fibre sensory neuropathy was also less severe in diabetic $i \mathrm{Nos}^{-/-}$than in wild-type mice.

Conclusions/interpretation iNOS plays a key role in peroxynitrite injury to peripheral nerve, and functional and structural changes of diabetic neuropathy. Nitrosative stress in axons and Schwann cells, rather than dorsal root ganglion neurons, underlies peripheral nerve dysfunction and degeneration.

Keywords iNOS · Nerve conduction · Nitrosative stress . Peripheral diabetic neuropathy - Tactile allodynia .

Thermal algesia

$\begin{array}{ll}\text { Abbreviations } \\ \text { DAB } & \text { 3,3'-diaminobenzidine } \\ \text { DRG } & \text { dorsal root ganglion } \\ \text { INFD } & \text { intraepidermal nerve fibre density } \\ \text { iNOS } & \text { inducible nitric oxide synthase } \\ \text { MNCV } & \text { motor nerve conduction velocity } \\ \text { NT } & \text { nitrotyrosine } \\ \text { PAR } & \text { poly(ADP-ribose) } \\ \text { PARP } & \text { poly(ADP-ribose) polymerase } \\ \text { PDN } & \text { peripheral diabetic neuropathy } \\ \text { PGP 9.5 } & \text { protein gene product 9.5 } \\ \text { SNCV } & \text { sensory nerve conduction velocity } \\ \text { STZ } & \text { streptozotocin } \\ \text { TBS } & \text { TRIS-buffered saline }\end{array}$

\section{Introduction}

Diabetic distal symmetric sensorimotor polyneuropathy affects $\sim 50 \%$ of patients with diabetes mellitus, and is a leading cause of foot amputation [1]. Evidence for the important role of the highly reactive oxidant peroxynitrite 
$[2,3]$ in peripheral diabetic neuropathy (PDN) is emerging from both experimental [4-7] and clinical [8-10] studies. Accumulation of nitrotyrosine (NT), a footprint of peroxynitrite injury, has been found in peripheral nerve, vasa nervorum, spinal cord and dorsal root ganglion (DRG) neurons in animal models of both type 1 (insulindependent) and type 2 (non-insulin-dependent) diabetes [3-7, 11-13] and high glucose-exposed cultured human Schwann cells [14]. Recent experimental studies with peroxynitrite decomposition catalysts revealed an important contribution of peroxynitrite-induced injury (so called nitrosative stress) to diabetes-induced motor and sensory nerve conduction deficits, small sensory nerve fibre dysfunction and degeneration, and autonomic neuropathy [3-7, 15]. Clinical studies revealed increased plasma NT levels and their correlation with endothelial dysfunction and redistribution of sudomotor responses, an early sign of sympathetic nerve dysfunction, in type 1 diabetic patients [8-10]. Furthermore, plasma peroxynitrite generation assessed by the pholasin chemiluminescence test correlated with the diabetic neuropathy impairment score of the lower limbs [10].

Peroxynitrite is a product of superoxide anion radical reaction with nitric oxide. The latter can be produced by several nitric oxide synthase isoforms, i.e. endothelial nitric oxide synthase, inducible nitric oxide synthase (iNOS), and neuronal nitric oxide synthase. Whereas all three isoforms have been localised in the peripheral nervous system [14, 16, 17], their individual contributions to peroxynitriteinduced injury in tissue targets for PDN and development of nerve fibre dysfunction and degeneration remain unknown. Here, we provide the first evidence of the key role of iNOS in diabetes-induced nitrosative stress in the peripheral nerve, nerve conduction deficit, and small-fibre sensory neuropathy.

\section{Methods}

\section{Reagents}

Unless otherwise stated, all chemicals were of reagentgrade quality, and were purchased from Sigma Chemical Company, St Louis, MO, USA. Rabbit polyclonal anti-NT antibody was purchased from Upstate (Lake Placid, NY, USA) and mouse monoclonal anti-poly(ADP-ribose) (PAR) from Trevigen (Gaithersburg, MD, USA). Secondary Alexa Fluor 488 goat anti-rabbit and Alexa Fluor 488 goat antimouse antibodies as well as Prolong Gold Antifade Reagent were purchased from Invitrogen (Eugene, OR, USA). Avidin/Biotin Blocking Kit, M.O.M. Basic Kit, VECTASTAIN Elite ABC Kit (Standard*), DAB Substrate Kit, and 3,3'-diaminobenzidine (DAB) were obtained from Vector
Laboratories (Burlingame, CA, USA). Rabbit polyclonal anti-protein gene product 9.5 (PGP 9.5) (ubiquitin Cterminal hydrolase) antibody was purchased from Chemicon International (Temecula, CA, USA). Other reagents for immunohistochemistry were purchased from Dako Laboratories (Santa Barbara, CA, USA).

Animals and limitations of the model of mice with iNos gene deficiency

The experiments were performed in accordance with regulations specified by the National Institutes of Health 'Principles of Laboratory Animal Care, 1985 Revised Version' and the Pennington Biomedical Research Center Protocol for Animal Studies. Several breeding pairs of B6.129P2-Nos2 $2^{\text {tmlLau} / \mathrm{J}}\left(\mathrm{iNos}^{-/-}\right)$mice, C57B16/J background, were obtained from Jackson Laboratories (Bar Harbor, ME, USA; stock number 002609). The use of $i \mathrm{Nos}^{-/}$mice for neurological studies is associated with some limitations because iNos gene (also known as Nos2) deficiency is known to result in impaired spinal cord regeneration, abnormal oligodendrocyte morphology and increased demyelination after neurotoxicant treatment (http://jaxmice.jax.org/strain/002609.html). Nevertheless, $i$ Nos $^{-1-}$ mice have normal motor and sensory nerve conduction velocities (MNCV and SNCV), normal thermal and mechanical algesia and tactile response thresholds, and, for this reason, have been considered suitable for assessment of the role of iNOS in PDN.

For the main experiment, a colony of $\mathrm{iNos}^{-/-}$mice was established at Pennington Biomedical Research Center. Mature male C57B16/J mice were purchased from Jackson Laboratories and served as controls. All the mice were fed standard mouse chow (PMI Nutrition International, Brentwood, MO, USA) and had access to water ad libitum. Diabetes was induced by a single injection of streptozotocin (STZ), $100 \mathrm{mg} \mathrm{kg}^{-1} \mathrm{day}^{-1}$, i.p., to non-fasted animals. Blood samples for glucose measurements were taken from the tail vein 3 days after STZ injection and the day before the animals were killed. The mice with blood glucose $\geq 13.8 \mathrm{mmol} / 1$ were considered diabetic. The injected mice that had blood glucose concentrations in the non-diabetic range were given low-dose STZ injections (40 $\mathrm{mg} \mathrm{kg}^{-1} \mathrm{day}^{-1}$, i.p.) until they developed hyperglycaemia (typically, one to three additional injections). At the end of the study (duration of diabetes of 6 weeks), the physiological and behavioural tests were performed in the following order: tactile responses to flexible von Frey filaments (first day), tail-pressure Randall-Sellito test (second day), thermal algesia by tailflick test (third day), thermal algesia by paw withdrawal test (fourth day), thermal algesia by hot plate test (fifth day), SNCV and MNCV (sixth day). Measurements of 
MNCV and SNCV were performed in mice anaesthetised with a mixture of ketamine and xylazine $(45 \mathrm{mg} / \mathrm{kg}$ body weight and $15 \mathrm{mg} / \mathrm{kg}$ body weight, respectively, i.p.).

Anaesthesia, killing and tissue sampling

The animals were sedated by $\mathrm{CO}_{2}$, and immediately killed by cervical dislocation. Sciatic nerves, DRG and foot pads were fixed in $10 \%$ neutral buffered formalin solution containing 4\% (wt/vol.) formaldehyde (Signa-Aldrich, Saint Louis, MO, USA) for assessment of NT and PAR by immunofluorescence histochemistry and intraepidermal nerve fibre density by conventional immunohistochemistry. PAR abundance is a measure of PAR polymerase (PARP) activity $[18,19]$.

Specific methods

Physiological tests Sciatic MNCV and hindlimb digital SNCV were measured as we have described elsewhere [3, 20]. A TCAT-2 Temperature Controller with a RET-3 Temperature probe and an HL-1 Heat Lamp (Physitemp Instruments, Clifton, NJ, USA) was used to maintain body and hindlimb temperature at $37^{\circ} \mathrm{C}$.

Behavioural tests Tactile responses were evaluated by quantifying the withdrawal threshold of the hind paw in response to stimulation with flexible von Frey filaments as we have described [13]. Tail pressure thresholds were registered with a Paw/Tail Pressure Analgesia meter for the Randall-Selitto test (37215; Analgesy-Meter, UGOBasile, Comerio VA, Italy). Pressure increasing at a linear rate of $10 \mathrm{~g}$ with the cut-off of $250 \mathrm{~g}$ to avoid tissue injury, was applied to the base of the tail. The applied tail pressure that evoked biting or licking behaviour was registered by an analgesia meter and expressed in grams. Three tests separated by at least 15 min were performed for each animal, and the mean value of these tests was calculated.

For thermal algesia, the paw withdrawal latency in response to the radiant heat $(15 \%$ intensity, which produced a heating rate of $\sim 1.3^{\circ} \mathrm{C} / \mathrm{s}$, cut-off time $30 \mathrm{~s}$ ) was determined as we have described [5-7, 12, 13] using an IITC model 336 TG combination tail-flick and paw algesia meter (IITC Life Science, Woodland Hills, CA, USA) with a floor temperature $\sim 32-33^{\circ} \mathrm{C}$ (manufacturer's set up). For assessment of tail-flick response latencies, the device was set at $40 \%$ heating intensity (heating rate $\sim 2.5^{\circ} \mathrm{C} / \mathrm{s}$ ) with a cut-off at $10 \mathrm{~s}$. In the hot plate test (IITC Model 39 Hot Plate Analgesia Meter; IITC Life Science) the unit had a plate preset temperature of $55^{\circ} \mathrm{C}$. In all three tests, at least three readings per animal were taken at 15 min interval, and the average was calculated.
Immunohistochemical studies All sections were processed by a single investigator and evaluated blindly. Low-power observations of skin sections stained for PGP 9.5 were made using a Zeiss Axioskop microscope (Carl Zeiss Imaging, Thornwood, NY, USA). Colour images were captured with a Zeiss Axiocam HRc CCD camera at $1,300 \times 1,030$ resolution (Carl Zeiss Imaging). Low-power images were generated with a $\times 40$ acroplan objective using the automatic capturing feature of the Zeiss Axiovision software (Ver. 3.1.2.1). Low-power observations of sciatic nerve and DRG sections stained for NT and PAR were made using a Zeiss Axioplan 2 imaging microscope. Colour images were captured with a Photometric CoolSNAP ${ }_{\mathrm{HQ}}$ CCD camera (Photometrics Sales, Tucson, AZ, USA) at $1,392 \times 1,040$ resolution. Low-power images were generated with a $\times 40$ acroplan objective using RS Image 1.9.2 software (Photometrics Sales).

NT immunoreactivities in the sciatic nerve and DRG were assessed by immunofluorescence histochemistry. In brief, sections were deparaffinised in xylene, rehydrated in decreasing concentrations of ethanol and washed in water. For immunofluorescence histochemistry, rabbit polyclonal anti-NT antibody was used in a working dilution of 1:400. Primary antibody was omitted in negative controls. Secondary Alexa Fluor 488 goat antirabbit antibody was applied in a working dilution of 1:200. Sections were mounted in Prolong Gold Antifade Reagent. The intensity of fluorescence was quantified using ImageJ 1.32 software (National Institutes of Health, Bethesda, MD, USA) and expressed as mean \pm SEM for each experimental group.

PAR immunoreactivity was assessed as described [20] with minor modifications. In brief, sections of peripheral nerve and DRG were deparaffinised in xylene, rehydrated in decreasing concentrations of ethanol and washed in water. Non-specific binding was blocked with the mouse Ig blocking reagent supplied with the Vector M.O.M. Basic Immunodetection Kit. Then mouse monoclonal anti-PAR antibody was diluted $1: 100$ in $1 \%$ BSA in TRIS-buffered saline (TBS), and applied overnight at $4^{\circ} \mathrm{C}$ in the humidity chamber. Primary antibody was omitted in negative controls. Secondary Alexa Fluor 488 goat anti-mouse antibody was diluted 1:200 in TBS and applied for $2 \mathrm{~h}$ at room temperature. Sections were mounted in Prolong Gold Antifade Reagent. At least ten fields of each section were examined to select one representative image. Representative images were microphotographed. The number of nuclei with identifiable PAR fluorescence was calculated for each microphotograph and expressed as mean \pm SEM for each experimental group. To evaluate neuronal PAR accumulation, a percentage of DRG neurons with weak, moderate and intense PAR immunofluorescence was calculated for each experimental group. 
Intraepidermal nerve fibre density (INFD) was assessed as described $[5,6,12,13]$. Three randomly chosen $5 \mu \mathrm{m}$ sections from foodpad skin of each mouse were deparaffinised in xylene, hydrated in decreasing concentrations of ethanol, and washed in water. Non-specific binding was blocked by $10 \%$ goat serum containing $1 \%$ BSA in TBS (DAKO, Carpinteria, CA, USA) for $2 \mathrm{~h}$, and the Avidin/ Biotin Blocking kit, according to the manufacturer's instructions. Then, rabbit polyclonal anti-PGP 9.5 antibody was applied in a 1:2,000 dilution. Secondary biotinylated goat anti-rabbit $\operatorname{IgG}(\mathrm{H}+\mathrm{L})$ antibody was applied in a 1:400 dilution, and the staining performed with the VECTASTAIN Elite ABC Kit (Standard*). For visualisation of specific binding sites, the DAB Substrate Kit containing DAB was used. Sections were counterstained with Gill's haematoxylin, dehydrated and mounted in Micromount mounting medium (Surgipath Medical, Richmond, IL, USA). Intraepidermal nerve fibre profiles were counted blindly by three independent investigators, under an Olympus BX-41 microscope (Leeds Precision Instruments, Minneapolis, MN, USA), and the average values were used. Microphotographs of stained sections were taken on an Axioscop 2 microscope (Zeiss) at $\times 4$ magnification, and the length of epidermis was assessed with the ImagePro 3.0 program (Media Cybernetics, Bethesda, MD, USA). An average of $2.8 \pm 0.3 \mathrm{~mm}$ of the sample length was investigated to calculate a number of nerve fibre profiles per mm of epidermis.

\section{Statistical analysis}

Results are expressed as means \pm SEM. Data were subjected to equality of variance $F$ test, and then to $\log _{10}$ transformation, if necessary, before one-way ANOVA. Where overall significance $(p<0.05)$ was attained, individual between-group comparisons were made using the Student-Newman-Keuls multiple-range test. Significance was defined at $p \leq 0.05$. When between-group variance differences could not be normalised by log transformation (datasets for body weights and plasma glucose), the data were analysed by the non-parametric Kruskal-Wallis oneway ANOVA, followed by the Bonferroni-Dunn test for multiple comparisons.

\section{Results}

Weight gain during the 6 week study was comparable in non-diabetic wild-type $(17 \%)$ and non-diabetic $i_{\mathrm{Nos}^{-/}}$ $(20 \%)$ mice (Table 1$)$. Whereas diabetic wild-type mice lost $14 \%$ of their initial body weight, diabetic $i \mathrm{Nos}^{-/}$mice did not display any weight loss, and moreover, gained $5 \%$ of weight. Initial (after STZ injection) blood glucose concentrations were $72 \%$ and $79 \%$ higher in diabetic wild-type and diabetic $i \mathrm{Nos}^{-1}$ mice compared with the corresponding controls. Hyperglycaemia progressed with the prolongation of diabetes, and the difference between final blood glucose concentrations in both groups and corresponding controls exceeded threefold. Final blood glucose concentrations were similar in diabetic wild-type and diabetic $\mathrm{iNos}^{-1-}$ mice.

Sciatic MNCV and hindlimb SNCV were 20 and $14 \%$ lower in diabetic wild-type mice compared with nondiabetic controls $(p<0.01$ for both comparisons, Fig. 1a, b). In contrast, diabetic $i \mathrm{Nos}^{-/-}$mice preserved normal MNCV and SNCV.

The latency of hind-paw withdrawal in response to radiant heat was increased by $103 \%$ in diabetic wild-type mice compared with the control group $(p<0.01)$, consistent with clearly manifest thermal hypoalgesia (Fig. 2a). This is in agreement with the results of tail-flick and hot plate tests, which also revealed increased thermal response latencies in the diabetic wild-type mice (Fig. 2b,c). In contrast, only very minor hypoalgesia (a 10\% increase in the response latency) was registered with the hind-paw withdrawal test in diabetic ${i N o s^{-/}}^{-1}$ mice. Note, however, that tail-flick response latencies were similarly increased in diabetic wildtype (21\%) and diabetic $i \mathrm{Nos}^{-/-}(17 \%)$ mice compared with the corresponding non-diabetic controls. The hot plate test results were in the normal range in diabetic $\mathrm{iNos}^{-1-}$ mice.

Table 1 Initial and final body weights and blood glucose concentrations in experimental groups

\begin{tabular}{|c|c|c|c|c|}
\hline \multirow[t]{2}{*}{ Group } & \multicolumn{2}{|c|}{ Body weight (g) } & \multicolumn{2}{|c|}{ Blood glucose $(\mathrm{mmol} / \mathrm{l})$} \\
\hline & Initial & Final & Initial & Final \\
\hline Non-diabetic wild-type & $28.6 \pm 0.9$ & $31.4 \pm 0.7$ & $8.6 \pm 0.4$ & $9.0 \pm 0.4$ \\
\hline Non-diabetic $i \mathrm{Nos}^{-/-}$ & $26.2 \pm 0.7$ & $32.1 \pm 1.0$ & $8.2 \pm 0.3$ & $9.1 \pm 0.4$ \\
\hline Diabetic wild-type & $30.6 \pm 0.8$ & $26.2 \pm 0.8 * *$ & $15.3 \pm 0.9^{* *}$ & $29.3 \pm 1.15^{* *}$ \\
\hline Diabetic $i \mathrm{Nos}^{-/}$ & $25.6 \pm 0.6$ & $26.8 \pm 0.4 * *$ & $19.1 \pm 1.1 * *$ & $27.9 \pm 1.5^{* *}$ \\
\hline
\end{tabular}

Data are means \pm SEM, $n=8-11$ per group

Significantly different from the corresponding non-diabetic groups $(* * p<0.01)$ 
a

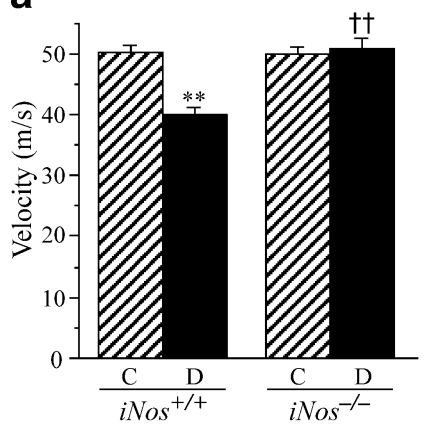

b

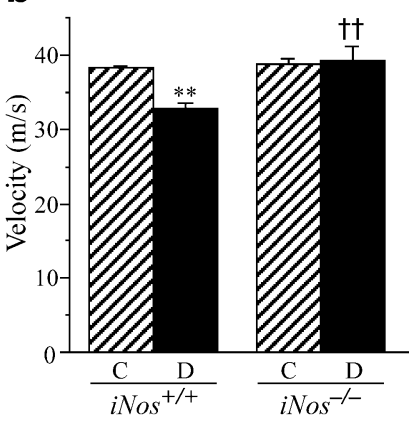

Fig. 1 Sciatic MNCV (a) and hindlimb digital sensory nerve conduction velocities (b) in control (C) and diabetic (D) wild-type and $i \mathrm{Nos}^{-/}$mice. Means \pm SEM, $n=6-8$ per group. ${ }^{* *} p<0.01$ vs corresponding non-diabetic groups; ${ }^{\dagger \dagger} p<0.01$ vs diabetic wild-type mice

Diabetic wild-type and $\mathrm{iNos}^{-1-}$ mice displayed moderate mechanical hypoalgesia detected with the tail pressure Randall-Sellito test (Fig. 3a). The tail pressure threshold was increased by $19 \%$ in diabetic wild-type mice and by $13 \%$ in diabetic $i N o s^{-1-}$ mice compared with the corresponding non-diabetic groups $(p<0.05$ and $p>0.05$, respectively). The severity of tactile allodynia was lower in diabetic wild-type compared with diabetic $\mathrm{iNos}^{-/-}$mice (Fig. 3b). Tactile response thresholds were reduced by $70 \%$ in diabetic wild-type mice and by $40 \%$ in diabetic $i \mathrm{Nos}^{-/}$ mice compared with the corresponding untreated groups ( $p$ $<0.01$ for both comparisons).

INFD was reduced by $36 \%$ in diabetic wild-type mice and by $14 \%$ in diabetic $\mathrm{iNos}^{-/-}$mice compared with the corresponding controls $(p<0.05$ and $p>0.05$, respectively, Fig. 4a,b). Note, however, that iNos gene deficiency was associated with a $\sim 10 \%$ decrease in INFD in non-diabetic mice.

NT immunofluorescence was increased by $69 \%$ in the sciatic nerves of diabetic wild-type mice compared with non-diabetic controls $(p<0.01$, Fig. 5a,b). In contrast, no diabetes-induced NT accumulation was detected in the

\section{a}

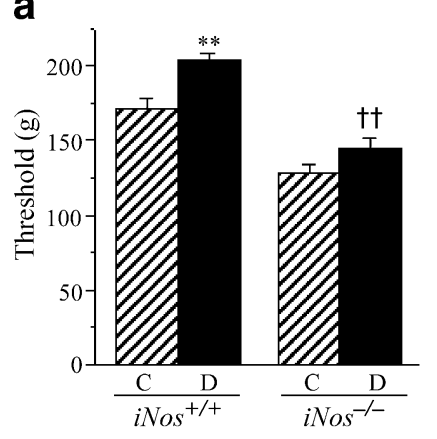

b

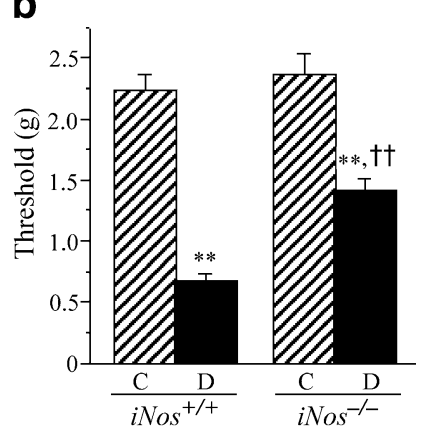

Fig. 3 Mechanical withdrawal thresholds in tail-pressure RandallSellito tests (a) and tactile response thresholds in response to stimulation with flexible von Frey filaments (b) in control (C) and diabetic (D) wild-type and $i \mathrm{Nos}^{-\digamma}$ mice. Means \pm SEM, $n=6-11$ per group. ${ }^{* *} p<0.01$ vs non-diabetic control mice; ${ }^{\dagger \dagger} p<0.01$ vs diabetic wild-type mice

sciatic nerves of $\mathrm{NNos}^{-/-}$mice. NT immunofluorescence of DRG was increased by $56 \%$ in diabetic wild-type mice and by $39 \%$ in diabetic $i \mathrm{Nos}^{-1-}$ mice compared with the corresponding non-diabetic groups $(p<0.01$ for both comparisons, Fig. 5c,d).

The number of PAR-positive nuclei was $71 \%$ higher in the sciatic nerve of diabetic wild-type mice, compared with non-diabetic controls (Fig. 6a,b). The percentage of DRG neurons with weak PAR immunofluorescence was lower, and of those with moderate and intense immunofluorescence higher in diabetic wild-type mice compared with the corresponding control group (Fig. 6c,d). iNos gene deficiency reduced the percentage of neurons with moderate and intense PAR fluorescence and increased the percentage of neurons with weak PAR fluorescence in diabetic mice.

\section{Discussion}

Evidence for the important role of iNOS in diabetic complications is emerging. Diabetes-associated iNOS upre-

a

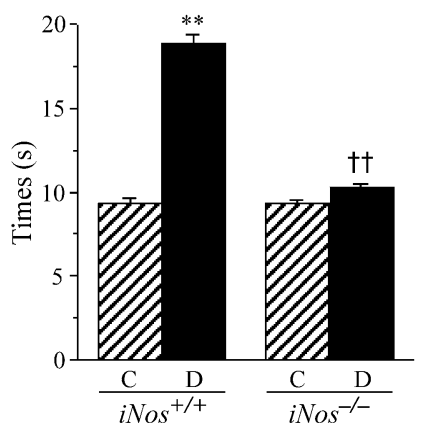

b

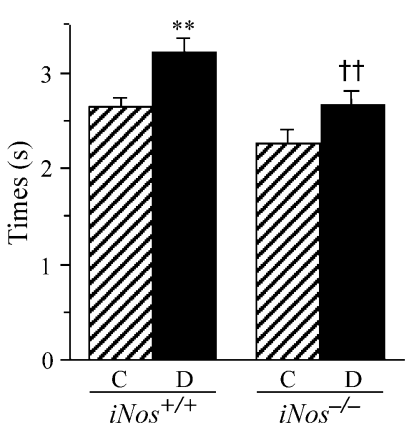

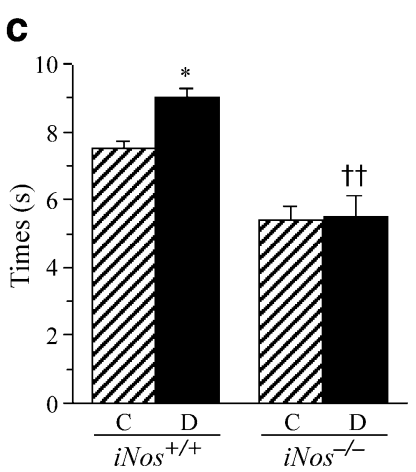

Fig. 2 Paw withdrawal latencies in response to radiant heat (a), tailflick test response latencies (b) and hot-plate test response latencies (c) in control (C) and diabetic (D) wild-type and $i \mathrm{Nos}^{-/}$mice. Means \pm
SEM, $n=8-11$ per group. ${ }^{*} p<0.05$ and ${ }^{* *} p<0.01$ vs corresponding non-diabetic groups; ${ }^{\dagger \dagger} p<0.01$ vs diabetic wild-type mice 

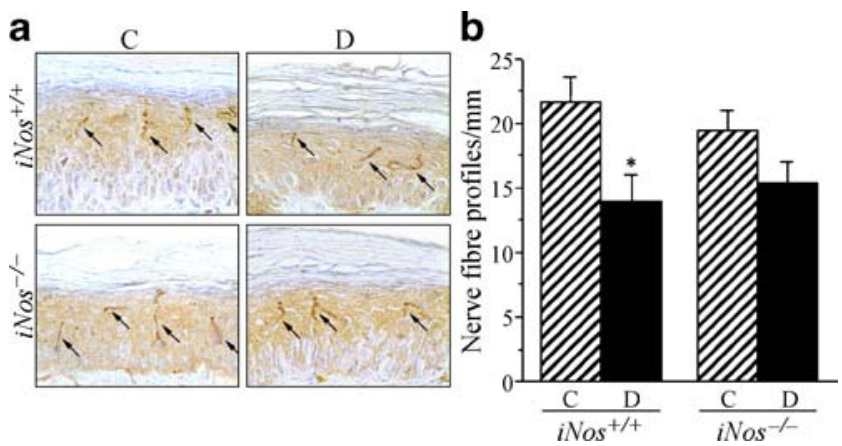

Fig. 4 Intraepidermal nerve fibre profiles in control (C) and diabetic (D) wild-type and $\mathrm{iNos}^{-/}$mice. a Representative images of intraepidermal nerve fibre profiles, magnification $\times 200$. Arrows indicate intra-epidermal nerve fibres. b Skin fibre density of control and diabetic wild-type and $i \mathrm{Nos}^{-/-}$mice. Means \pm SEM, $n=8-11$ per group. ${ }^{*} p<0.05$ vs control mice

gulation has been found in the retina [21], heart [22], vascular endothelium [23] and smooth muscle layer [24]. Furthermore, high glucose-induced iNOS overexpression has been reported for human Schwann cells [14], human retinal and coronary artery endothelial cells $[25,26]$, and rat and murine glomerular mesangial cells $[27,28]$. Advanced glycation end-products induced iNos expression via a p38
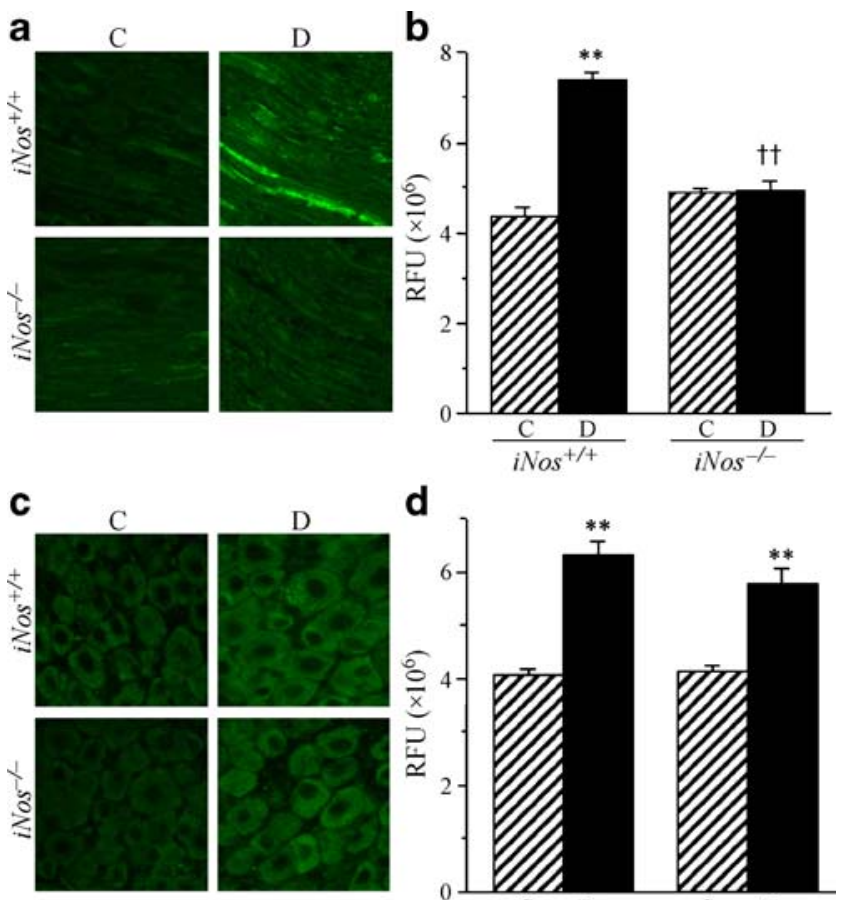

d

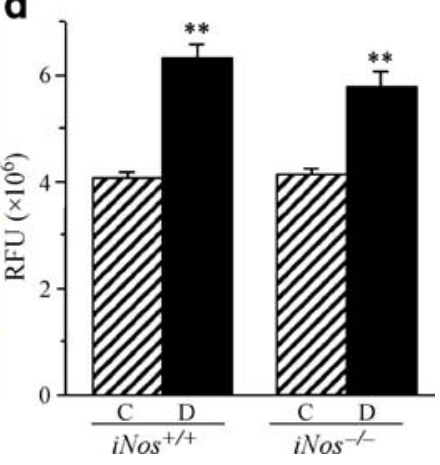

Fig. 5 Representative microphotographs of immunofluorescent staining of NT in sciatic nerves (a) and DRG (c) of control (C) and diabetic (D) wild-type and $\mathrm{iNos}^{-1}$ mice. Magnification $\times 40$. NT fluorescence counts in sciatic nerves (b) and DRG (d) of control and diabetic wildtype and $\mathrm{iNos}^{-/-}$mice. Means \pm SEM, $n=7-11$ per group. ${ }^{* *} p<0.01$ vs non-diabetic control mice; ${ }^{\dagger \dagger} p<0.01$ vs diabetic wild-type mice. RFU, relative fluorescence units a
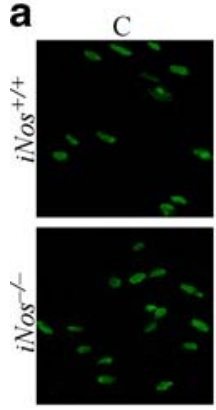

(1)

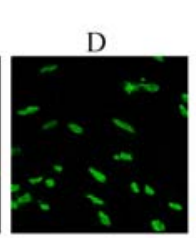

b
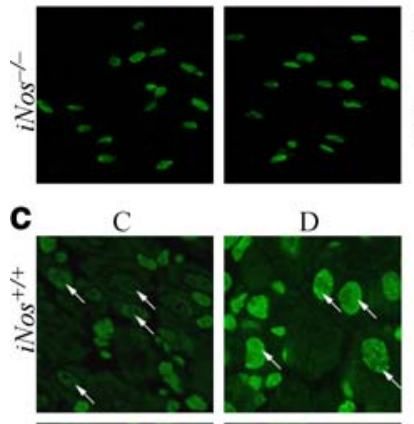

D
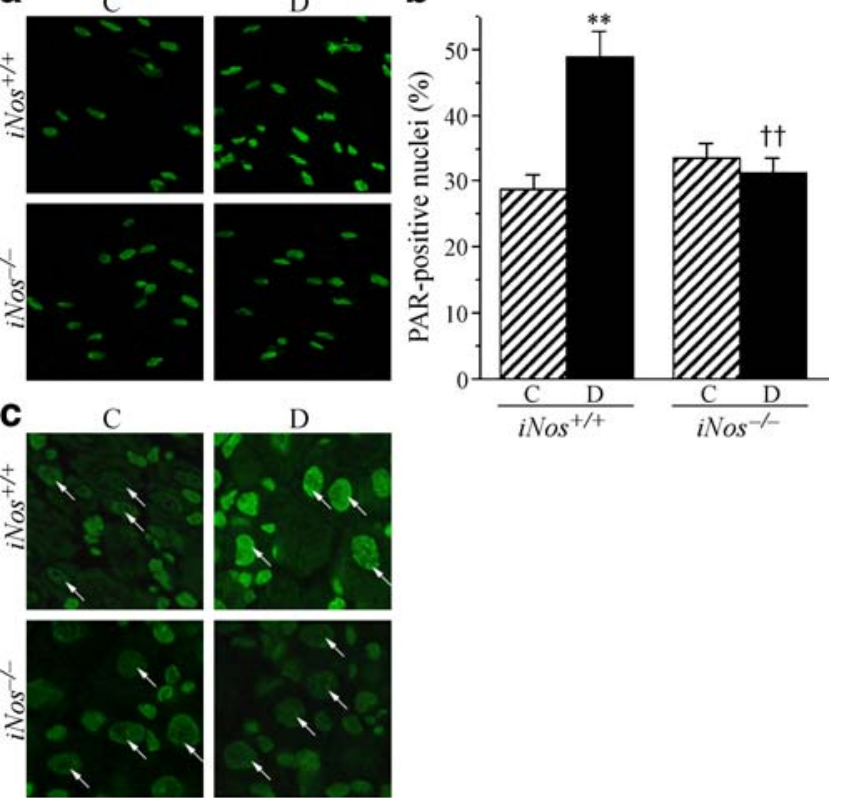

d

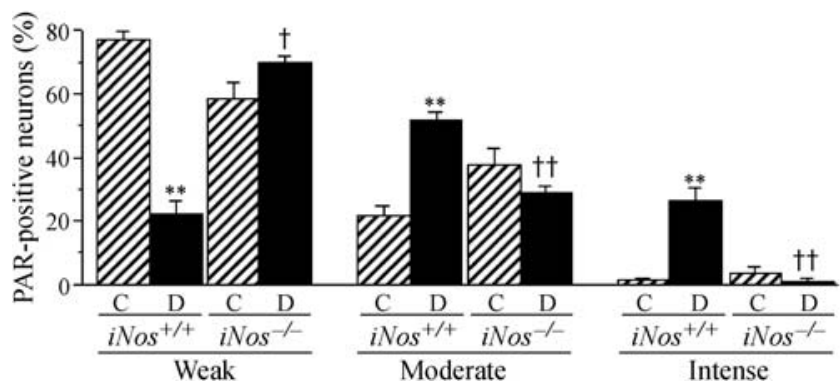

Fig. 6 Representative microphotographs of immunofluorescent staining of PAR in sciatic nerve (a) and DRG neurons (c) of control (C) and diabetic (D) wild-type and $i \mathrm{Nos}^{-/}$mice. Magnification $\times 40$. (b) The number of PAR-positive nuclei in sciatic nerve; $\mathbf{d}$ percentage of DRG neurons with weak, moderate, and intense PAR immunofluorescence. The number of DRG neurons with weak, moderate and intense PAR immunofluorescence was expressed as a percentage of neurons with identifiable PAR immunofluorescence (examples are shown by white arrows) in the dorsal root ganglia of control and diabetic wild-type and $i \mathrm{Nos}^{-/}$mice. Means \pm SEM, $n=8-11$ per group. ${ }^{* *} p<0.01$ vs non-diabetic control mice; ${ }^{\dagger \dagger} p<0.01$ vs diabetic wild-type mice

mitogen-activated protein kinase-dependent pathway [29]. PARP activation, another important player in the regulation of iNos gene expression, upregulated iNOS via nuclear factor-kappaB activation [30]. Studies in $\mathrm{iNos}^{-/-}$mice revealed an important role for iNOS in subnormal retinal oxygenation, leucostasis, blood-renal barrier breakdown and formation of pericyte ghosts and acellular capillaries characteristic of peripheral diabetic retinopathy [31-33]. They have also implicated iNOS in diabetes-induced endothelial dysfunction [23], impaired vascular reactivity [24], cardiomyopathy [34], myocardial ischaemia-reperfusion injury [35] and stroke [36], as well as glomeruloscle- 
rosis and tubulointerstitial fibrosis characteristic of chronic diabetic nephropathy [37].

The role of iNOS in diabetic neuropathy has not been studied. iNos gene expression has been identified in rat peripheral nerves and dorsal root ganglia [16]. iNos mRNA expression was found to be reduced rather than increased in the sciatic nerve of rat models of both short-term and longterm diabetes [16]. The latter is consistent with the demonstration of a diabetes-associated decrease in iNos mRNA expression in penile intracavernous nerves [17]. Nevertheless, the present study unequivocally demonstrates a key role of iNOS in peroxynitrite injury to peripheral nerve, motor and sensory nerve conduction deficits, and small-fibre sensory neuropathy.

Our findings are consistent with other reports suggesting that nitrosative stress is a characteristic feature of experimental PDN [38-40]. Accumulation of NT is clearly manifest in peripheral nerve and DRG neurons of wildtype STZ-diabetic mice, consistent with previous observations of our group and others made in STZ-diabetic rodents $[4,6,7]$, as well as in $o b / o b$ [12] and high-fat-diet fed [13] mice. Using specific markers for certain cell types of PNS, we localised immunoreactive NT in endothelial and Schwann cells of peripheral nerve, as well as neuronal and glial cells of dorsal root ganglia (V. R. Drel and I. G. Obrosova, unpublished results). Thus, nitrosative stress affects all major tissue targets for PDN. Diabetic $i \mathrm{Nos}^{-/-}$ mice did not display NT accumulation in peripheral nerve, but were not protected from nitrosative stress in DRG. Despite the latter circumstance, $\mathrm{iNos}^{-/}$mice preserved normal MNCV and SNCV. Furthermore, iNos gene deficiency alleviated the severity of small-fibre sensory neuropathy. These findings suggest that iNOS-dependent peroxynitrite formation in axons and Schwann cells, rather than cell bodies, of peripheral nerve plays a major role in functional and structural changes of diabetic neuropathy.

Evidence for the important role of PARP activation, another phenomenon closely linked to oxidative-nitrosative stress, in diabetic complications is emerging [18, 41, 42]. We [20, 43-45] and others [15] have demonstrated a key role of PARP activation in motor and sensory nerve conduction and nerve blood flow deficits, thermal hyper-and hypoalgesia, mechanical hyperalgesia, tactile allodynia, exaggerated flinching behaviour in the formalin pain test, and small sensory nerve fibre degeneration associated with PDN, as well as in diabetic autonomic neuropathy. PARP activation manifest by PAR accumulation was present in both sciatic nerve and DRG in diabetic wild-type mice. iNos gene deficiency completely prevented diabetes-induced peripheral nerve PAR accumulation, consistent with the lack of enhanced nitrosative stress. Of interest, despite the presence

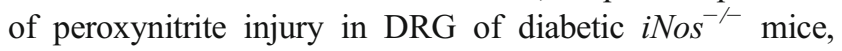
PAR accumulation in DRG neurons was markedly alleviat- ed. The significance and mechanisms of this effect cannot be interpreted based on current knowledge. However, growing evidence suggests that PARP activation is not a mere consequence of oxidative-nitrosative stress, but can be mediated via other biochemical mechanisms, e.g. phosphorylation by extracellular signal regulated kinase [46].

In conclusion, iNOS plays a key role in peroxynitrite injury to peripheral nerve, MNCV and SNCV deficits and small-fibre sensory neuropathy. Nitrosative stress in axons and Schwann cells, rather than DRG neurons, plays a major role in peripheral nerve dysfunction and degeneration associated with PDN. The findings support the rationale for development of specific inhibitors of $i$ Nos for prevention and treatment of this devastating complication of diabetes mellitus.

Acknowledgements The study was supported by the American Diabetes Association Research Grant 7-05-RA-102, the Juvenile Diabetes Research Foundation International Grant 1-2005-223, and the National Institutes of Health Grant DK 071566-01 (all to I. G. Obrosova).

Duality of interest The authors declare that there is no duality of interest associated with this manuscript.

\section{References}

1. Boulton AJ, Vinik AI, Arezzo JC et al (2005) American Diabetes Association. Diabetic neuropathies: a statement by the American Diabetes Association. Diabetes Care 28:956-962

2. Pacher P, Beckman JS, Liaudet L (2007) Nitric oxide and peroxynitrite in health and disease. Physiol Rev 87:315-424

3. Szabó C, Ischiropoulos H, Radi R (2007) Peroxynitrite: biochemistry, pathophysiology and development of therapeutics. Nat Rev Drug Discov 6:662-680

4. Obrosova IG, Mabley JG, Zsengeller Z et al (2005) Role for nitrosative stress in diabetic neuropathy: evidence from studies with a peroxynitrite decomposition catalyst. FASEB J 19:401-403

5. Vareniuk I, Pavlov IA, Drel VR et al (2007) Nitrosative stress and peripheral diabetic neuropathy in leptin-deficient $(\mathrm{ob} / \mathrm{ob})$ mice. Exp Neurol 205:425-436

6. Drel VR, Pacher P, Vareniuk I et al (2007) Evaluation of the peroxynitrite decomposition catalyst Fe(III) tetra-mesitylporphyrin octasulfonate on peripheral neuropathy in a mouse model of type 1 diabetes. Int J Mol Med 20:783-792

7. Obrosova IG, Drel VR, Oltman CL et al (2007) Role of nitrosative stress in early neuropathy and vascular dysfunction in streptozotocin-diabetic rats. Am J Physiol Endocrinol Metab 293:E1645E1655

8. Hoeldtke RD (2003) Nitrosative stress in early type 1 diabetes. David H. P. Streeten Memorial Lecture. Clin Auton Res 13:406-421

9. Ceriello A, Piconi L, Esposito K, Giugliano D (2007) Telmisartan shows an equivalent effect of vitamin $\mathrm{C}$ in further improving endothelial dysfunction after glycemia normalization in type 1 diabetes. Diabetes Care 30:1694-1698

10. Nourooz-Zadeh J, Ziegler D, Sohr C, Betteridge JD, Knight J, Hothersall J (2006) The use of pholasin as a probe for the determination of plasma total antioxidant capacity. Clin Biochem 39:55-61 
11. Cheng C, Zochodne DW (2003) Sensory neurons with activated caspase-3 survive long-term experimental diabetes. Diabetes 52:2363-2371

12. Drel VR, Mashtalir N, Ilnytska O et al (2006) The leptin-deficient (ob/ob) mouse: a new animal model of peripheral neuropathy of type 2 diabetes and obesity. Diabetes 55:3335-3343

13. Obrosova IG, Ilnytska O, Lyzogubov VV et al (2007) High-fat diet induced neuropathy of pre-diabetes and obesity: effects of 'healthy' diet and aldose reductase inhibition. Diabetes 56:2598-2608

14. Obrosova IG, Drel VR, Pacher P et al (2005) Oxidativenitrosative stress and poly(ADP-ribose) polymerase (PARP) activation in experimental diabetic neuropathy: the relation is revisited. Diabetes 54:3435-3441

15. Nangle MR, Cotter MA, Cameron NE (2004) Effects of the peroxynitrite decomposition catalyst, FeTMPyP, on function of corpus cavernosum from diabetic mice. Eur J Pharmacol 502:143-148

16. Zochodne DW, Verge VM, Cheng C et al (2000) Nitric oxide synthase activity and expression in experimental diabetic neuropathy. J Neuropathol Exp Neurol 59:798-807

17. El-Sakka AI, Lin CS, Chui RM, Dahiya R, Lue TF (1999) Effects of diabetes on nitric oxide synthase and growth factor genes and protein expression in an animal model. Int $\mathrm{J}$ Impot Res 11:123-132

18. Garcia Soriano F, Virag L, Jagtap P et al (2001) Diabetic endothelial dysfunction: the role of poly(ADP-ribose) polymerase activation. Nat Med 7:108-113

19. Jagtap P, Szabo C (2005) Poly(ADP-ribose) polymerase and the therapeutic effects of its inhibitors. Nat Rev Drug Discov 4: 421-440

20. Obrosova IG, Li F, Abatan OI et al (2004) Role of poly(ADPribose) polymerase activation in diabetic neuropathy. Diabetes 53:711-720

21. Ellis EA, Guberski DL, Hutson B, Grant MB (2002) Time course of NADH oxidase, inducible nitric oxide synthase and peroxynitrite in diabetic retinopathy in the BBZ/WOR rat. Nitric Oxide 6:295-304

22. Jesmin S, Zaedi S, Maeda S, Yamaguchi I, Goto K, Miyauchi T (2006) Effects of a selective endothelin a receptor antagonist on the expressions of iNOS and eNOS in the heart of early streptozotocin-induced diabetic rats. Exp Biol Med (Maywood) 231:925-931

23. Nagareddy PR, Xia Z, McNeill JH, MacLeod KM (2005) Increased expression of iNOS is associated with endothelial dysfunction and impaired pressor responsiveness in streptozotocin-induced diabetes. Am J Physiol Heart Circ Physiol 289: H2144-H2152

24. Ishikawa T, Kohno F, Kawase R, Yamamoto Y, Nakayama K (2004) Contribution of nitric oxide produced by inducible nitric oxide synthase to vascular responses of mesenteric arterioles in streptozotocin-diabetic rats. $\mathrm{Br} \mathrm{J}$ Pharmacol 141:269-276

25. Steinle JJ (2007) Sympathetic neurotransmission modulates expression of inflammatory markers in the rat retina. Exp Eye Res 84:118-125

26. Rajesh M, Mukhopadhyay P, Bátkai S et al (2007) Cannabidiol attenuates high glucose-induced endothelial cell inflammatory response and barrier disruption. Am J Physiol Heart Circ Physiol 293:H610-H619

27. Noh H, Ha H, Yu MR et al (2002) High glucose increases inducible NO production in cultured rat mesangial cells. Possible role in fibronectin production. Nephron 90:78-85

28. Sharma K, Danoff TM, DePiero A, Ziyadeh FN (1995) Enhanced expression of inducible nitric oxide synthase in murine macrophages and glomerular mesangial cells by elevated glucose levels: possible mediation via protein kinase C. Biochem Biophys Res Commun 207:80-88
29. Chang PC, Chen TH, Chang CJ, Hou CC, Chan P, Lee HM (2004) Advanced glycosylation end products induce inducible nitric oxide synthase (iNOS) expression via a p38 MAPKdependent pathway. Kidney Int 65:1664-1675

30. Ha HC, Hester LD, Snyder SH (2002) Poly(ADP-ribose) polymerase-1 dependence of stress-induced transcription factors and associated gene expression in glia. Proc Natl Acad Sci USA 99:3270-3275

31. Berkowitz BA, Luan H, Gupta RR et al (2004) Regulation of the early subnormal retinal oxygenation response in experimental diabetes by inducible nitric oxide synthase. Diabetes 53:173-178

32. Leal EC, Manivannan A, Hosoya K et al (2007) Inducible nitric oxide synthase isoform is a key mediator of leukostasis and blood-retinal barrier breakdown in diabetic retinopathy. Invest Ophthalmol Vis Sci 48:5257-5265

33. Zheng L, Du Y, Miller C et al (2007) Critical role of inducible nitric oxide synthase in degeneration of retinal capillaries in mice with streptozotocin-induced diabetes. Diabetologia 50:1987-1996

34. Crespo MJ, Zalacaín J, Dunbar DC, Cruz N, Arocho L (2008) Cardiac oxidative stress is elevated at the onset of dilated cardiomyopathy in streptozotocin-diabetic rats. J Cardiovasc Pharmacol Ther 13:64-71

35. Marfella R, Di Filippo C, Esposito K et al (2004) Absence of inducible nitric oxide synthase reduces myocardial damage during ischemia reperfusion in streptozotocin-induced hyperglycemic mice. Diabetes 53:454-462

36. Kitayama J, Faraci FM, Gunnett CA, Heistad DD (2006) Impairment of dilator responses of cerebral arterioles during diabetes mellitus: role of inducible NO synthase. Stroke 37:2129-2133

37. Trachtman H, Futterweit S, Pine E, Mann J, Valderrama E (2002) Chronic diabetic nephropathy: role of inducible nitric oxide synthase. Pediatr Nephrol 17:20-29

38. Coppey LJ, Gellett JS, Davidson EP, Dunlap JA, Lund DD, Yorek MA (2001) Effect of antioxidant treatment of streptozotocininduced diabetic rats on endoneurial blood flow, motor nerve conduction velocity, and vascular reactivity of epineurial arterioles of the sciatic nerve. Diabetes 50:1927-1937

39. Oltman CL, Davidson EP, Coppey LJ et al (2008) Vascular and neural dysfunction in Zucker diabetic fatty rats: a difficult condition to reverse. Diabetes Obes Metab 10:64-74

40. Ho EC, Lam KS, Chen YS et al (2006) Aldose reductase-deficient mice are protected from delayed motor nerve conduction velocity, increased c-Jun NH2-terminal kinase activation, depletion of reduced glutathione, increased superoxide accumulation, and DNA damage. Diabetes 55:1946-1953

41. Zheng L, Szabó C, Kern TS (2004) Poly(ADP-ribose) polymerase is involved in the development of diabetic retinopathy via regulation of nuclear factor-kappaB. Diabetes 53:2960-2967

42. Szabó C, Biser A, Benko R, Böttinger E, Suszták K (2006) Poly (ADP-ribose) polymerase inhibitors ameliorate nephropathy of type 2 diabetic Leprdb/db mice. Diabetes 55:3004-3012

43. Li F, Szabó C, Pacher P et al (2004) Evaluation of orally active poly (ADP-ribose) polymerase inhibitor in streptozotocin-diabetic rat model of early peripheral neuropathy. Diabetologia 47:710-717

44. Ilnytska O, Lyzogubov VV, Stevens MJ et al (2006) Poly(ADPribose) polymerase inhibition alleviates experimental diabetic sensory neuropathy. Diabetes 55:1686-1694

45. Obrosova IG, Xu W, Lyzogubov VV et al (2008) PARP inhibition or gene deficiency counteracts intraepidermal nerve fiber loss and neuropathic pain in advanced diabetic neuropathy. Free Radic Biol Med 44:972-981

46. Kauppinen TM, Chan WY, Suh SW, Wiggins AK, Huang EJ, Swanson RA (2006) Direct phosphorylation and regulation of poly(ADP-ribose) polymerase-1 by extracellular signal-regulated kinases 1/2. Proc Natl Acad Sci USA 103:7136-7141 